\title{
Polarized Raman Spectra of Oriented Films of $\alpha$-Helical Poly(L-alanine) and its $N$-Deuterated Analogue
}

Sang-Ho Lee and Samuel Krimm*

Biophysics Research Division and Department of Physics, University of Michigan, Ann Arbor, Michigan 48109, USA

\begin{abstract}
Polarized Raman spectra were obtained on well oriented films of $\alpha$-helical poly(L-alanine) ( $\alpha$-PLA) and its $N$ deuterated analogue in order to obtain definitive assignments of the $E_{2}$-species modes. Such assignments were aided by an analysis of factors that can lead to 'cross-talk' between $A, E_{1}$ and $E_{2}$ spectra, viz. reflection of the incident beam and non-perfect lateral and axial chain orientation in the sample. The convincing assignment of at least eleven significant $E_{2}$ modes in $\alpha$-PLA has led to the refinement of a new empirical force field and a more detailed normal mode analysis. (C) 1998 John Wiley \& Sons, Ltd.
\end{abstract}

J. Raman Spectrosc. 29, 73-80 (1998)

\section{INTRODUCTION}

Poly(L-alanine) (PLA) is the simplest L-type polypeptide, with a methyl group as its side chain. It is well known that this molecule can take on a well defined right-handed $\alpha$-helix or an antiparallel-chain pleated $\beta$ sheet, depending on the sample preparation method. Much attention has been paid to this molecule as a first step in understanding the structures and functions of polypeptides and proteins. In this effort there have been extensive studies of its infrared ${ }^{1-8}$ and Raman ${ }^{3,9-15}$ spectra and several normal mode calculations on $\alpha$-PLA have also been done. ${ }^{4,16-19}$

In general, the vibrational normal modes of an infinite regular helix are characterized by $A, E_{1}$ and $E_{2}$ symmetry species, ${ }^{20,21}$ where $A$ and $E_{1}$ species are infrared active and $A, E_{1}$ and $E_{2}$ species are Raman active. For a complete vibrational analysis of $\alpha$-helical PLA, it is essential to separate all three symmetry species experimentally. Separation of $A$ and $E_{1}$ species is usually possible through polarized infrared radiation with an oriented sample, and extensive work on $\alpha$-PLA was done over 40 years ago by Elliott. ${ }^{1}$ Since the $E_{2}$-species modes are optically active only in Raman spectra, the separation of the $E_{2}$ - from the $A$ - and $E_{1}$-species modes is indispensable for a complete vibrational analysis. About 30 years ago, Fanconi et al. ${ }^{9}$ obtained the explicit forms of Raman tensors corresponding to the optically active Raman $A, E_{1}$ and $E_{2}$ modes, and partially succeeded in separating each symmetry species by using polarized laser Raman scattering. However, owing to difficulties in preparing an oriented sample, there has

* Correspondence to: S. Krimm, Biophysics Research Division and Department of Physics, University of Michigan, Ann Arbor, Michigan 48109, USA.

Contract grant sponsor: NSF

Contract grant number: MCB-9115906;

Contract grant number: DMR-911053. been no further advance in polarized Raman studies on $\alpha$-PLA.

We have been able to obtain good polarized Raman spectra of oriented films of $\alpha$-PLA and its $N$-deuterated analogue. Polarized infrared and far-infrared spectra of such (essentially uniaxial) oriented films have also been measured, ${ }^{22}$ and we have been able to separate convincingly vibrational modes belonging to each of the three symmetry species. Based on these new experimental results, a new empirical force field has been refined and a rigorous normal mode analysis has been done for $\alpha$ PLA. ${ }^{22}$

We report here the results of the polarized Raman studies on oriented $\alpha$-PLA and its $N$-deuterated analogue ( $\alpha$-PLA-ND). The detailed vibrational analysis will be presented elsewhere. ${ }^{22}$

\section{SELECTION RULES FOR A REGULAR HELICAL POLYPEPTIDE}

The Raman activities of a molecule can be determined from non-zero projections of the polarizability tensor, $\alpha$, on each symmetry species, viz. the Raman tensors: if the projection to a particular symmetry species has any non-zero tensor element then a vibrational transition belonging to that symmetry species is Raman active in its fundamental. ${ }^{23}$ For an infinite regular helical polymer there are in general three Raman tensors: ${ }^{9}$

$$
\begin{aligned}
{[\alpha]_{A} } & =\frac{1}{2}\left(\begin{array}{ccc}
\alpha_{11}+\alpha_{22} & \alpha_{12}-\alpha_{21} & 0 \\
\alpha_{21}-\alpha_{12} & \alpha_{11}+\alpha_{22} & 0 \\
0 & 0 & 2 \alpha_{33}
\end{array}\right) \\
{[\alpha]_{E_{1}} } & =\frac{1}{2}\left(\begin{array}{ccc}
0 & 0 & \alpha_{13}-i \alpha_{23} \\
0 & 0 & \alpha_{23}+i \alpha_{13} \\
\alpha_{31}-i \alpha_{32} & \alpha_{32}+i \alpha_{31} & 0
\end{array}\right)
\end{aligned}
$$




$$
[\alpha]_{E_{2}}=\frac{1}{4}\left(\begin{array}{cc}
\alpha_{11}-\alpha_{22}-i\left(\alpha_{12}+\alpha_{21}\right) & \\
\alpha_{12}+\alpha_{21}+i\left(\alpha_{11}-\alpha_{22}\right) & \\
0 & \\
\alpha_{12}+\alpha_{21}+i\left(\alpha_{11}-\alpha_{22}\right) & 0 \\
\alpha_{22}-\alpha_{11}+i\left(\alpha_{12}+\alpha_{21}\right) & 0 \\
0 & 0
\end{array}\right)
$$

where the helix axis is taken as the $z$-axis corresponding to the Cartesian coordinate index 3 and vibrational modes belonging to the $E_{1}$ and $E_{2}$ symmetry species are doubly degenerate with $[\alpha]_{E_{1^{*}}}=\left([\alpha]_{E_{1}}\right)^{*}$ and $[\alpha]_{E_{2^{*}}}=$ $\left([\alpha]_{E_{2}}\right)^{*}$, respectively. For a helical structure with an exact threefold rotational symmetry, as in an ideal $3_{10^{-}}$ helix, the $E_{1}$ and $E_{2}$ modes are degenerate in such a way that $[\alpha]_{E_{2}}=[\alpha]_{E_{1} *}$ and they are identical with each other.

In using Eqns (1)-(3) for an oriented helix, we can plausibly assume that the polarizability tensor $\alpha$ is symmetric and also satisfies

$$
\alpha_{11}=\alpha_{22} \neq \alpha_{33}
$$

since there is no preference between the $x$-axis and the $y$-axis. Under these assumptions, the above Raman tensors become

$$
\begin{aligned}
{[\alpha]_{A} } & =\left(\begin{array}{ccc}
\alpha_{11} & 0 & 0 \\
0 & \alpha_{11} & 0 \\
0 & 0 & \alpha_{33}
\end{array}\right) \\
{[\alpha]_{E_{1}} } & =\frac{1}{2}\left(\begin{array}{ccc}
0 & 0 & \alpha_{13}-i \alpha_{23} \\
0 & 0 & \alpha_{23}+i \alpha_{13} \\
\alpha_{31}-i \alpha_{32} & \alpha_{32}+i \alpha_{31} & 0
\end{array}\right) \\
{[\alpha]_{E_{2}} } & =\frac{1}{2}\left(\begin{array}{ccc}
-i \alpha_{12} & \alpha_{12} & 0 \\
\alpha_{12} & +i \alpha_{12} & 0 \\
0 & 0 & 0
\end{array}\right)
\end{aligned}
$$

These tensors can be used to separate all the optically active Raman modes of the $A, E_{1}$ and $E_{2}$ symmetry species for an oriented helical sample with perfect axial and lateral alignment. If we assume the helix axis (sample orientation direction) as the $z$-axis and the direction in which the scattered light is observed as the
Table 1. Experimental set-ups related to Fig. 1 and corresponding selection of symmetry species from polarized Raman spectra of oriented helical fibers

$\begin{array}{ccc}\text { Experimental set-up } & \\ \text { (a) } & (\mathrm{b}) & \text { Symmetry species } \\ Z(x x) Y-\alpha_{11} \mathrm{~b} & X(z z) Y-\alpha_{33} & A \\ Z(x z) Y-\alpha_{31} & X(z x) Y-\alpha_{13} \mathrm{a} & E_{1} \\ Z(y z) Y-\alpha_{32} & X(y z) Y-\alpha_{32} & E_{1} \\ Z(y x) Y-\alpha_{12} \mathrm{~b} & X(y x) Y-\alpha_{12} \mathrm{~b} & E_{2}\end{array}$

a Just for simplicity, this experimental arrangement was used in our $E_{1}$ spectral measurements. For the selection of $\alpha_{32}$ component $[X(y z) Y$ arrangement] a half-wave plate is required so as to change the polarization direction of incident light.

b In uniaxially oriented helical fibers, in which a random orientation exists about the helix axis, the spectrum from the $Z(x x) Y$ arrangement is essentially identical with those from $X\left(y_{x}\right) Y$ or $Z\left(y_{x}\right) Y$ arrangements (see text).

$y$-axis (Fig. 1), the experimental separation of the $A, E_{1}$ and $E_{2}$ species can be achieved as given in Table 1 Each experiment is designated in a particular way, e.g. $X(y x) Y ; X$ designates the direction in which the incident laser beam propagates and $Y$ denotes the direction in which the scattered light is observed; $y$ denotes the polarization direction of the incident laser beam and $x$ denotes that of the scattered light. Since the induced dipole moment, $\vec{\mu}^{\text {ind. }}$, due to the incident electric field, $\overrightarrow{\mathbf{E}}^{\text {inc. }}$, is given by

$$
\vec{\mu}_{j}^{\text {ind. }}=\sum_{k=1}^{3} \alpha_{j k} \vec{E}_{k}^{\text {inc. }}+\cdots
$$

$X(y x) Y$ is related to the $\alpha_{12}$ component and selects only the $E_{2}$ symmetry species through Eqn (7) by using the experimental set-up (b) (Fig. 1). Similarly, $X(z z) Y$ and $X(z x) Y$ select only $A$ and $E_{1}$ symmetry species, respectively. In the experimental set-up (a), it is impossible to place the oriented sample parallel to the incident laser beam direction, and usually the sample has to be tilted slightly, resulting in mixing between the spectra of $Z(x x) Y-\alpha_{11}$ and $Z(y x) Y-\alpha_{12}$. Therefore, the experimental set-up (b) was used in our spectral measurements, and in the foregoing discussion our $A, E_{1}$ and $E_{2}$

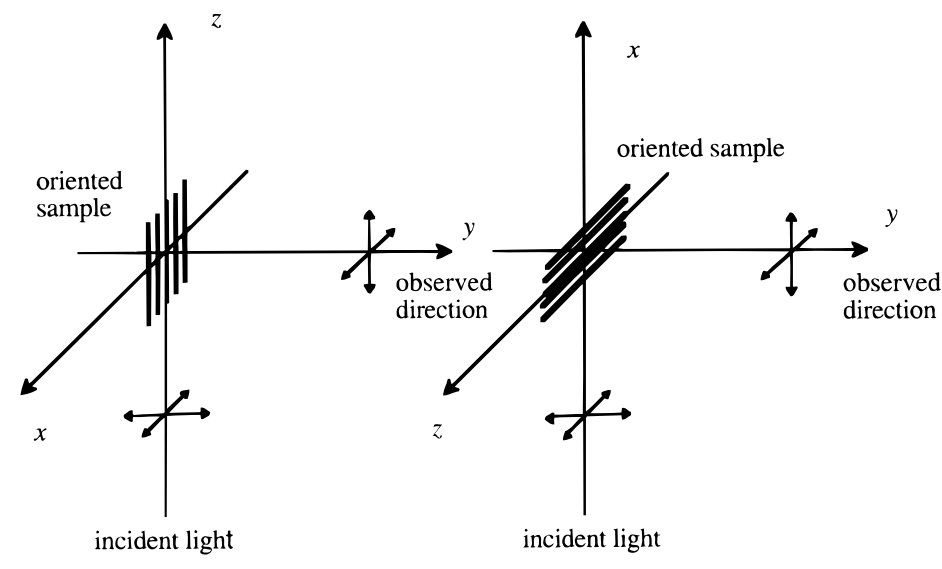

(a)

(b)

Figure 1. Schematic experimental set-up for polarized Raman spectra. Incident laser beam (a) parallel and (b) perpendicular to the oriented fiber axes. 
spectra represent $X(z z) Y, X(z x) Y$ and $X(y x) Y$ arrangements, respectively. Of course, if the helix axis orientation is not perfect, some 'cross-talk' between these spectra will occur (see below).

\section{EXPERIMENTAL}

The PLA samples used in this study were purchased from Sigma Chemical [molecular weight based on viscosity determination $\approx 23000$, degree of polymerization $(D P) \approx 324)]$. When the initially powdered sample is dissolved in dichloroacetic acid (DCA), it is well known $^{2}$ that the resulting PLA is in the right-handed $\alpha$-form. The concentrated solutions of PLA in DCA were injected into diethyl ether. The resulting precipitated cluster of swollen samples was kneaded on a glass plate by using a stainless-steel micro-spatula until it became fairly sticky. Oriented $\alpha$-PLA films were made by stroking this sticky sample with a smooth-edged fine metal piece. The kneading process was crucial to achieving a well oriented film by stroking. For the Raman spectra, oriented samples were made on a glass slide and sample films were separated later from the glass. The residual solvent (DCA) was removed from the film by extraction with diethyl ether or pure water followed by drying in a vacuum oven at $40^{\circ} \mathrm{C}$. During the extraction, the sample film usually separated by itself from the glass slide. For Raman spectra the sample was mounted again on a glass slide for easy sample holding.

Polarized infrared spectra ${ }^{22}$ showed a very high degree of orientation in such a film: for example, in the best samples the $908(A), 892\left(E_{1}\right) \mathrm{cm}^{-1}$ pair $^{19}$ were clearly separated, with barely detectable shoulders seen in the unexpected polarization, and similarly for the $1518(A), 1548\left(E_{1}\right) \mathrm{cm}^{-1}$ pair. ${ }^{19}$ The degree of orientation was less perfect for the somewhat thicker Raman samples but, as the spectra show, it was sufficiently high to permit a separation of the symmetry species.

The $N$-deuterated analogue of an oriented $\alpha$-PLA was made by using $O$-deuterated DCA. In order to increase the degree of $\mathrm{N}$-deuteration, oriented films made from $O$-deuterated DCA were soaked in $\mathrm{D}_{2} \mathrm{O}$ for about 1 day at $65^{\circ} \mathrm{C}$. The $O$-deuterated DCA was distilled from the mixed solution of $\mathrm{D}_{2} \mathrm{O}(95 \%)$ and undeuterated DCA $(5 \%)$. Distillation was repeated with the predominantly $O$-deuterated DCA a second time, and more than $95 \%$ of the DCA was $O$-deuterated.

Raman spectra were recorded using a Spex 1403 double monochromator with excitation by $514.5 \mathrm{~nm}$ radiation from an argon ion laser and $2 \mathrm{~cm}^{-1}$ resolution. Oriented film samples of reasonable thickness were mounted on a small glass slide (thickness $c a .1$ $\mathrm{mm}$ ). Data were obtained with an entrance slit size of $280 \mu \mathrm{m}$, an accumulation time of $1 \mathrm{~s}$ in each increment of $1 \mathrm{~cm}^{-1}$ and 60 scans at a laser power of $0.16 \mathrm{~W}$ for undeuterated samples (50 scans at a laser power of 0.15 $\mathrm{W}$ for the $N$-deuterated sample). Since the PLA sample prepared from DCA can easily be burnt, it is important to defocus the incident laser beam at the sample to an adequate extent. In order to reduce the background fluorescence, the sample had been exposed to a laser beam of $0.07 \mathrm{~W}$ for about $7-15 \mathrm{~h}$ before the actual data were recorded.

\section{RESULTS AND DISCUSSION}

The observed polarized Raman spectra of $\alpha$-PLA are shown in Fig. 2 and those of $\alpha$-PLA-ND in Fig. 3. The band wavenumbers, plus clear spectral species assignments, are listed in Table 2. Since the assignment of $A$ and $E_{1}$ modes is unambiguous from the polarized infrared spectra, ${ }^{22}$ we focus this discussion primarily on the identification of the $E_{2}$ modes.

In this connection, a few points need to be considered carefully to interpret the observed spectra correctly. First, a detailed look into the experimental arrangement $X(y x) Y$ (Fig. 4), which was used to record our $E_{2}$ spectra and was believed to select out only the $\alpha_{12}$ component, shows that this set-up can indeed additionally select out the $\alpha_{11}$ component corresponding to the $X(x x) Y$ arrangement, which can give rise to $A$-species bands through Eqn (5) due to the reflected light from the glass slide or from the back sample surface contacting the glass slide. The reflected light acts like another incident beam having some component polarized in the $x$-direction. The resulting appearance of $A$ bands from the $\alpha_{11}$ component, in addition to the dominant $E_{2}$ bands from $\alpha_{12}$, cannot be completely eliminated even though the glass slide holding the oriented sample is removed. As a result of this effect, a vibrational mode that can give an intense $A$-species Raman

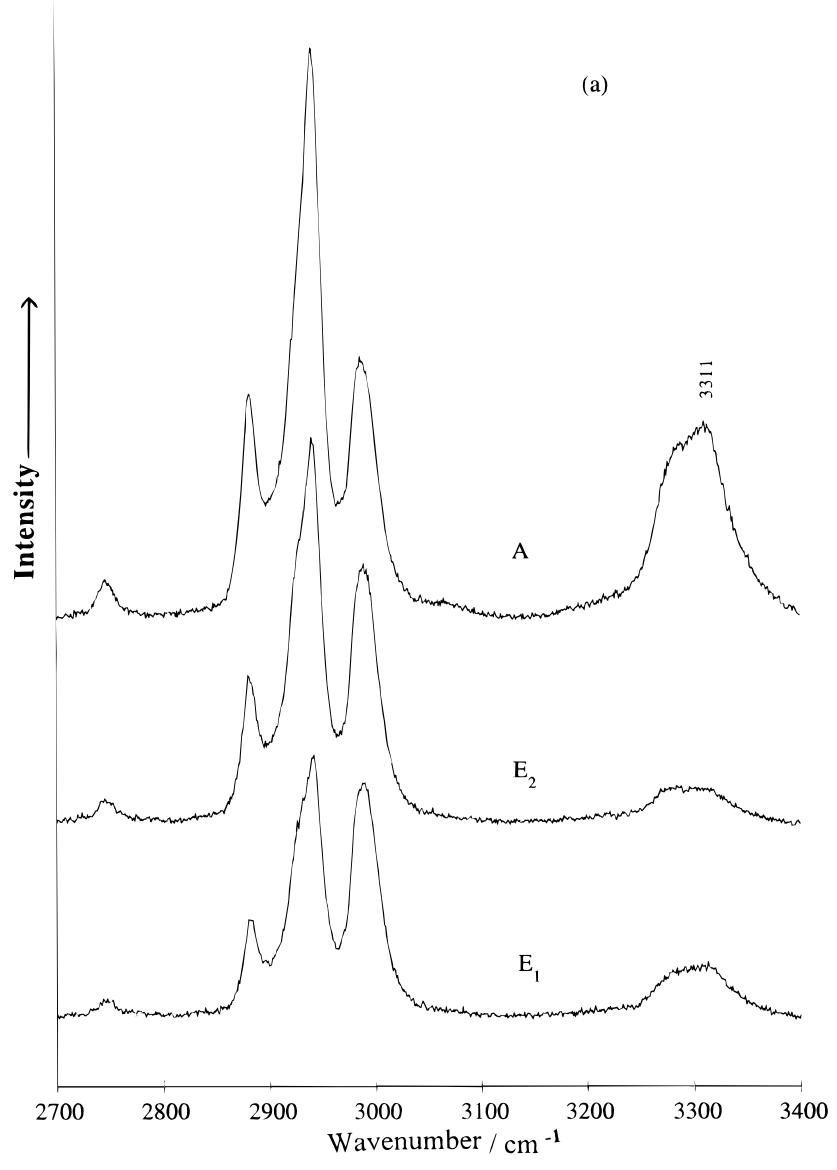

Figure 2. Polarized Raman spectra of oriented $\alpha$-PLA-NH $A=X(z z) Y, E_{1}=X(z x) Y$ and $E_{2}=X(y x) Y$ spectra. Intensity scale is arbitrary. (a) $2700-3400 \mathrm{~cm}^{-1}$ region; (b) $800-1800 \mathrm{~cm}^{-1}$ region; (c) $100-800 \mathrm{~cm}^{-1}$ region; (d) $30-100 \mathrm{~cm}^{-1}$ region. 


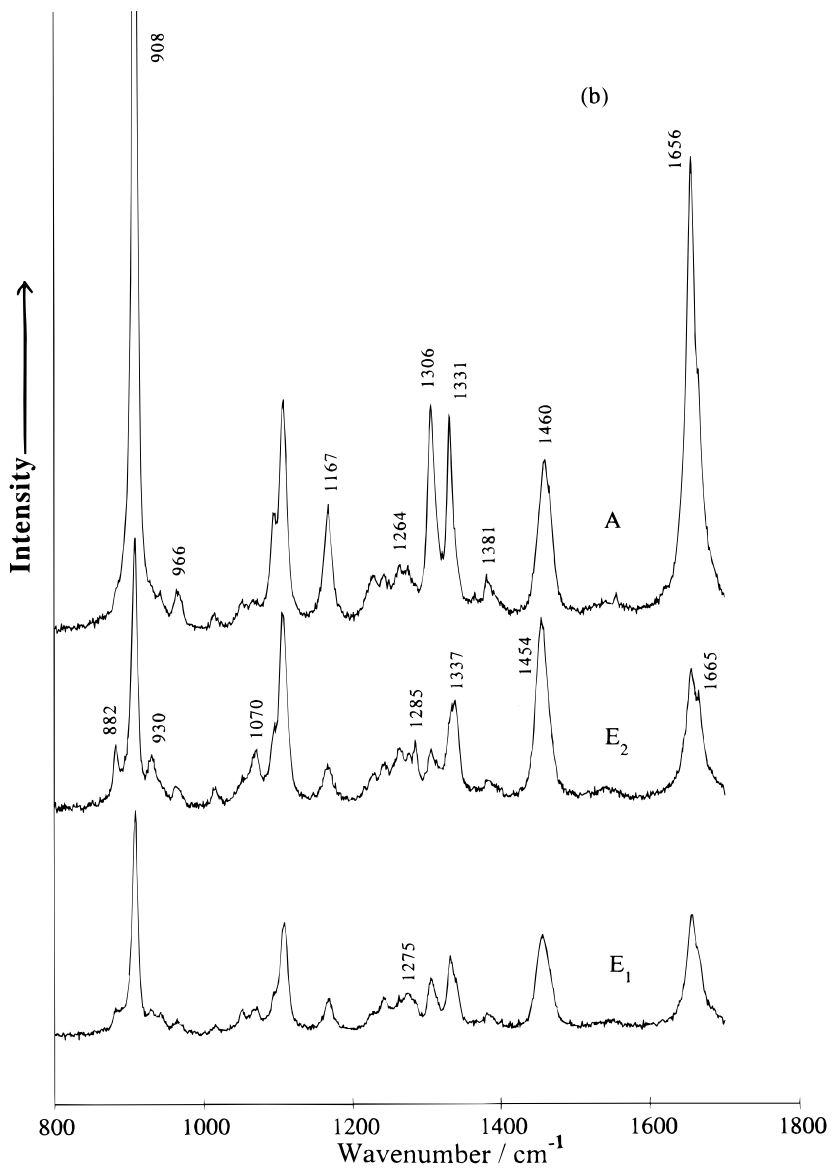

Figure 2. Continued

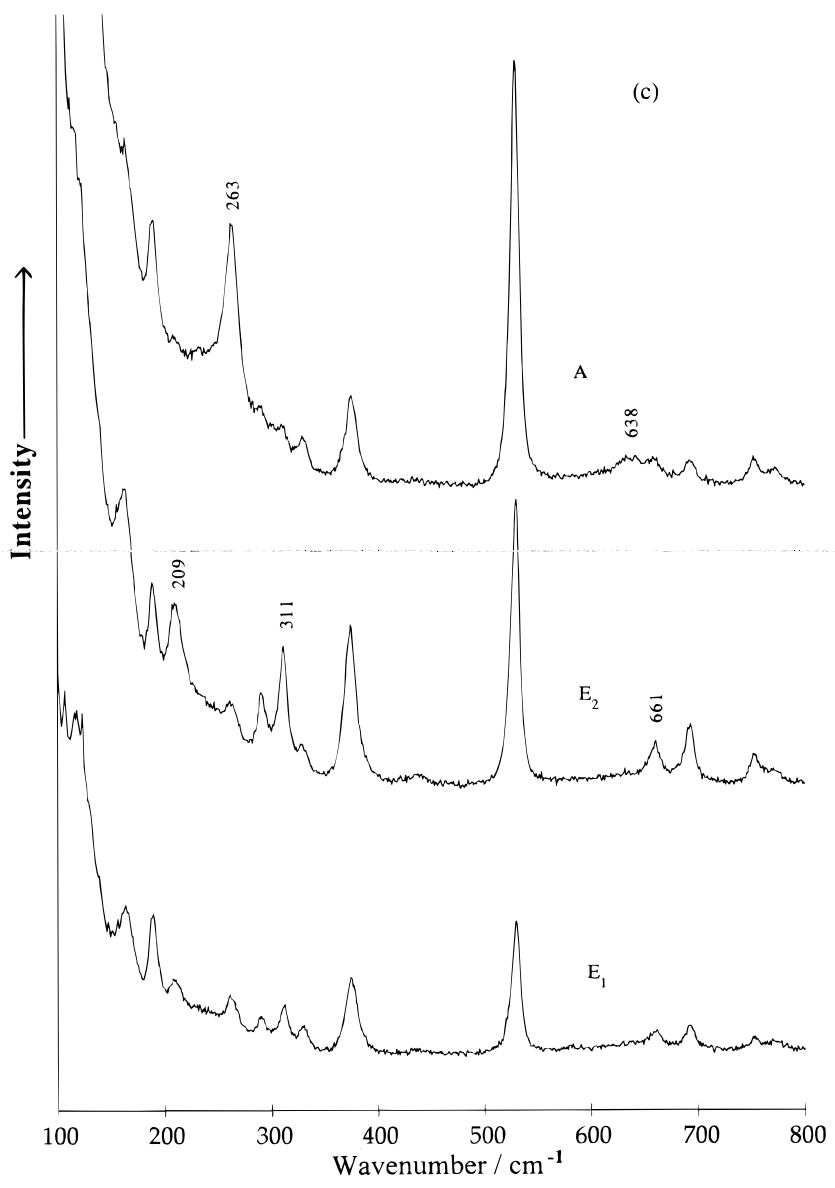

Figure 2. Continued

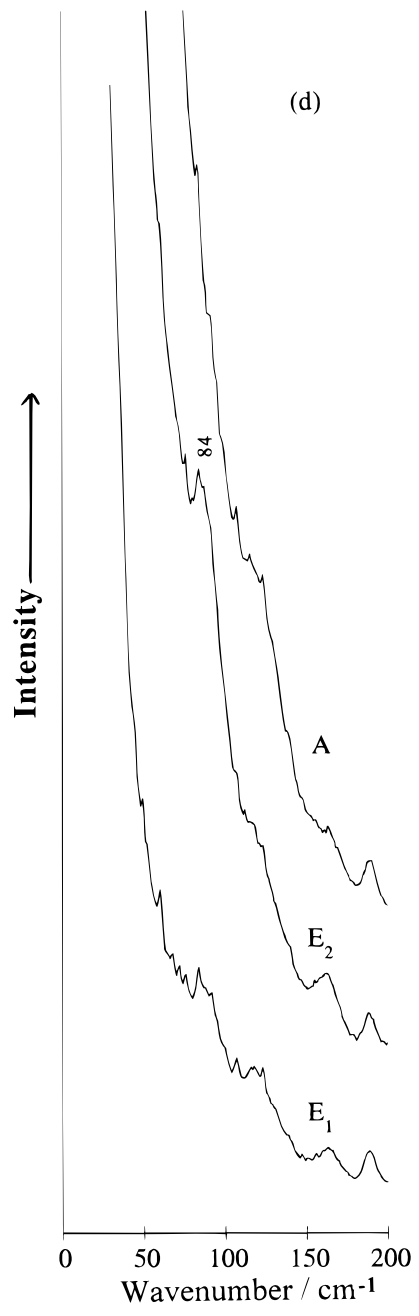

Figure 2. Continued

band from $\alpha_{11}$ can also appear with significant intensity in the $E_{2}$ spectra.

A more important problem arises from the fact that all helices do not have the same orientation about the helix axis, i.e. the sample orientation method only gives uniaxial orientation. This means that observationally $\alpha_{11}$ will be equivalent to $\alpha_{12}$. In fact, when we measured a $Z(x x) Y-\alpha_{11}(A)$ spectrum [using experimental set-up (a) and fixing the sample orientation axis $5-10^{\circ}$ from the incident beam], we found it to be the same as the $X(y x) Y-\alpha_{12}\left(E_{2}\right)$ spectrum. Therefore, in contrast to what was previously thought, ${ }^{9}$ in the case of a uniaxially oriented sample, i.e. with random orientation about the helix axis, we cannot independently separate modes due to $\alpha_{11}\left(A\right.$-species) from those due to $\alpha_{12}\left(E_{2}\right.$ species). However, for a uniaxially oriented sample those bands in the $E_{2}$ spectrum that have significantly different wavenumbers from corresponding bands in the $A\left(X(z z) Y-\alpha_{33}\right)$ and $E_{1}$ spectra can be confidently assigned to the $E_{2}$ species, while some $E_{2}$ bands of the same wavenumber as those in the $A$ spectrum might be due to $\alpha_{11}$.

Finally, we must consider the effect due to the nonperfect axial orientation in the sample. In order to give a reasonable Raman intensity the sample has to be fairly thick, and it is more difficult to obtain a high 


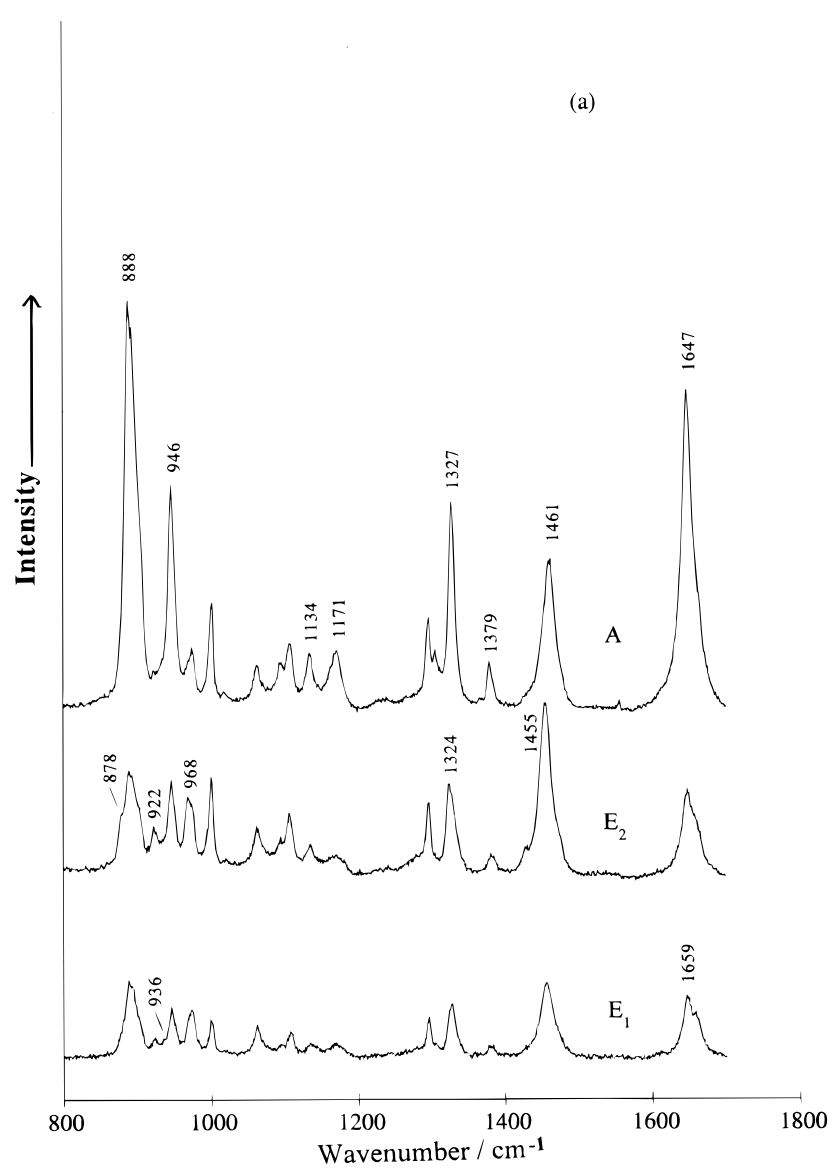

Figure 3. Polarized Raman spectra of oriented $\alpha$-PLA-ND: $A=X(z z) Y, E_{1}=X(z X) Y$ and $E_{2}=X(y X) Y$ spectra. Intensity scale is arbitrary. (a) $800-1800 \mathrm{~cm}^{-1}$ region; (b) $100-800 \mathrm{~cm}^{-1}$ region: (c) $30-100 \mathrm{~cm}^{-1}$ region.

degree of axial orientation in such a thick sample. Nevertheless, we still think that the majority of the PLA helix axes are parallel to the stroking direction. Such a small non-perfect orientation in the sample [where Eqn (4) no longer holds] leads to slight additional $E_{1}$ (from $\alpha_{13}$ or $\alpha_{31}$ ) spectral components in the $A$ spectra, $A$ (from $\alpha_{33}$ or $\alpha_{11}$ ) and $E_{2}$ (from $\alpha_{11}$; see Eqn (3)) spectral components in the $E_{1}$ spectra and $E_{1}\left(\right.$ from $\left.\alpha_{32}\right)$ spectral components in the $E_{2}$ spectra. Although the degree of orientation in the sample for Raman scattering is less than that in the sample for infrared, our polarized spectra overall show that the samples prepared by the stroking method are oriented well enough to separate some key bands belonging to each of the three symmetry species.

In the amide $A$ region we observe two NH stretch (s) bands centered around $3311 \mathrm{~ms}(A)$ and $3288 \mathrm{~m}(A) \mathrm{cm}^{-1}$, respectively. This doublet nature, which is more prominent in the infrared spectra, turned out to be dependent on the amount of residual solvent DCA in the oriented PLA sample. Detailed analysis of this region will be presented elsewhere, ${ }^{24}$ but it shows that the medium strong band around $3311(A) \mathrm{cm}^{-1}$ arises from the $\alpha$ helical structure while the band around $3288 \mathrm{~m}(A) \mathrm{cm}^{-1}$ is due to an additional structure in the PLA sample. Since the CH s modes $\left(3000-2800 \mathrm{~cm}^{-1}\right)$ can be involved in extensive Fermi resonances with $\mathrm{CH}$ bend

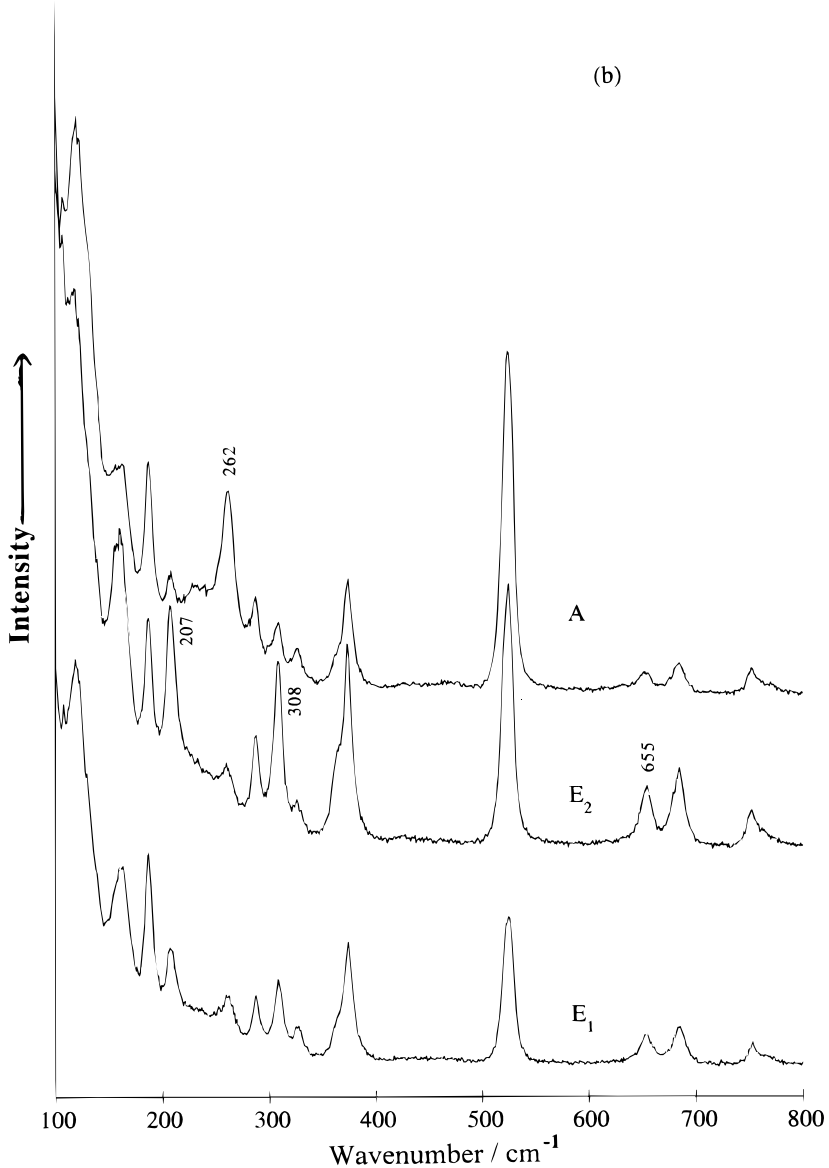

Figure 3. Continued

modes, ${ }^{25}$ they will not be discussed further here.

In addition to the typical amide I band at $1656 \mathrm{vs}(A)$ $\mathrm{cm}^{-1}$ [on $N$-deuteration at $1647 \mathrm{vs}(A) \mathrm{cm}^{-1}$ ], we observed a clear $E_{2}$ spectral band at $1665 \mathrm{mw} \mathrm{cm}^{-1}$ (no significant corresponding band could be seen in the polarized infrared spectrum). In view of the expectation that in this spectrum there will also be some $A$ mode contribution from the $\alpha_{11}$ component, the evident presence of the $1665 \mathrm{~cm}^{-1}$ band must be considered compelling, and we newly assign it to the $E_{2}$ amide I mode. Its wavenumber is significantly higher than the value of $1642 \mathrm{~cm}^{-1}$ previously predicted from a perturbation transition dipole coupling analysis, ${ }^{19}$ but is consistent with a treatment incorporating direct interaction force constants. $^{22}$

The strong $1454 \mathrm{~cm}^{-1}$ band in the $E_{2}$ spectrum, with a definite shoulder at $1460 \mathrm{~cm}^{-1}$ corresponding to the $A$-species mode, indicates that it should be assigned to an $E_{2}$-species mode. Similarly, the $1337 \mathrm{~m} \mathrm{~cm}^{-1}$ band in the $E_{2}$ spectrum is distinctly separable from the $1331 \mathrm{~s}(A) \mathrm{cm}^{-1}$ band, which is clearly an $A$-species mode from the infrared spectrum and whose contribution to the $E_{1}$ and $E_{2}$ spectra we have anticipated. We therefore assign the $1337 \mathrm{~cm}^{-1}$ band to the $E_{2}$ species.

The early assignment of the amide III modes by Chen and Lord $^{14}$ is well confirmed by the observed bands at $1264 \mathrm{mw}(A)$ and $1275 \mathrm{mw}\left(E_{1}\right) \mathrm{cm}^{-1}$. The clear presence of an $E_{2}$-spectral band at $1285 \mathrm{mw} \mathrm{cm}^{-1}$ fits such an assignment and is consistent with earlier normal mode 


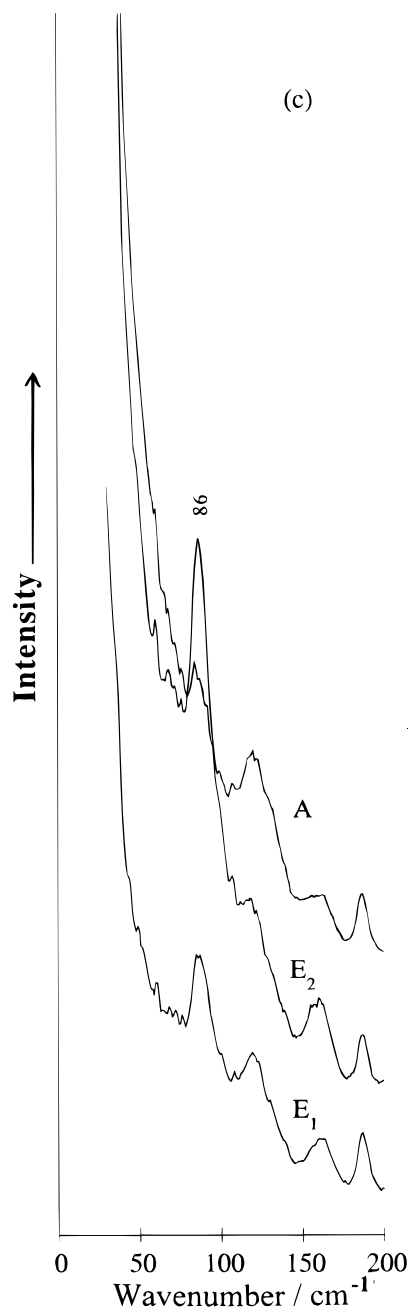

Figure 3. Continued

calculations of its expected position. ${ }^{19}$ The corresponding amide III' modes are observed at $946 \mathrm{~s}(A), 973 \mathrm{~m}\left(E_{1}\right)$ and $968 \mathrm{~m}\left(E_{2}\right) \mathrm{cm}^{-1}$ in the spectrum of $\alpha$-PLA-ND. The lower wavenumber of the $E_{2}$ mode compared with the
$E_{1}$ mode in this case is evident from the $E_{2}$ spectrum and is well reproduced by the newly refined force field. $^{22}$

The bands near $1106 \mathrm{~s} \mathrm{~cm}^{-1}$ in the $A, E_{1}$ and $E_{2}$ spectra may well be present in all three species, and normal mode calculations indeed show this to be the case. ${ }^{22}$ The $E_{2}$ spectrum, however, unambiguously shows that bands at $1070 \mathrm{mw}, 930 \mathrm{mw}$ and $882 \mathrm{mw} \mathrm{cm}^{-1}$ should be assigned exclusively to this species. The last two seem to have counterparts at $922 \mathrm{w}$ and $878 \mathrm{sh}$ $\mathrm{cm}^{-1}$, respectively, in the $E_{2}$ spectrum of $\alpha$-PLA-ND.

A few bands appear to present a problem in assignment to a species, in that they seem to be too strong in the $E_{2}$ spectrum in view of the fact that the normal mode calculations, ${ }^{22}$ and in some cases the polarized infrared spectra, place them in the $A$ species. Thus, the $752 \mathrm{mw}(A) \mathrm{cm}^{-1}$ band, while it could also be expected to show up from the experimental reflected beam, seems to be unusually strong in the $E_{2}$ spectrum. Of course, the significant presence in the $E_{2}$ spectrum of strong $A$ species bands at 1655 and $908 \mathrm{~cm}^{-1}$, where neither is expected from the calculation ${ }^{22}$ is consistent with this observation and may just indicate that these modes are associated with large $\alpha_{11}$ components. The $693 \mathrm{~cm}^{-1}$ band is clearly an $A$-species mode from the infrared spectrum ${ }^{19,22}$ and its appearance in the $E_{2}$ spectrum may be due to a similar effect. Note that all these bands have nearly the same wavenumbers in $A$ and $E_{2}$ spectra. The $661 \mathrm{mw} \mathrm{cm}^{-1}$ band clearly belongs to the $E_{2}$ species, as does its counterpart at $655 \mathrm{~m} \mathrm{~cm}^{-1}$ in $\alpha$-PLA-ND. This new information, together with the shift of the $693 \mathrm{~cm}^{-1}$ band to $685 \mathrm{~cm}^{-1}$ in $\alpha$-PLA-ND, justifies reassigning these bands to skeletal modes ${ }^{22}$ rather than the previous assignment of the $c a .660 \mathrm{~cm}^{-1}$ band to an amide $\mathrm{V}$ mode. ${ }^{19}$ The $638 \mathrm{mw}(A) \mathrm{cm}^{-1}$ band, which disappears on $N$-deuteration and was not previously noted, ${ }^{19}$ will also be seen ${ }^{22}$ to be assignable to the $A$-species amide $\mathrm{V}$ mode.

The 530 and $374 \mathrm{~cm}^{-1}$ bands could be present in all species. The $311 \mathrm{~ms} \mathrm{~cm}^{-1}$ band clearly belongs to the $E_{2}$ species, as do those at $209 \mathrm{~m}$ and $84 \mathrm{~m} \mathrm{~cm}^{-1}$. The 290

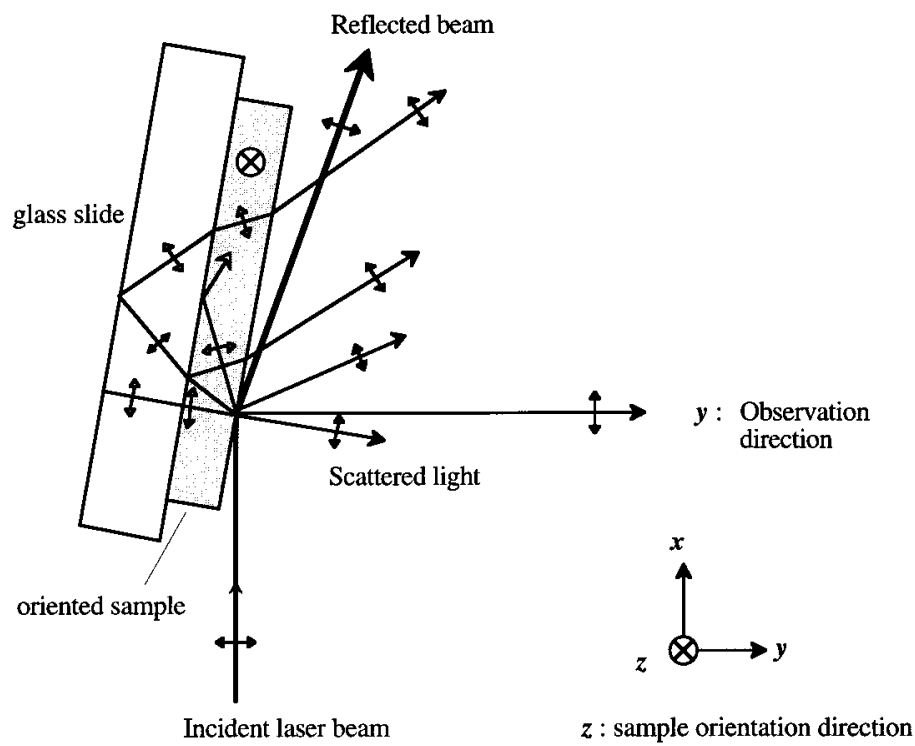

Figure 4. Schematic projection along the sample orientation direction of an experimental set-up for the polarized Raman $E_{2}$ spectra. The incident laser beam is polarized in the $y$-axis. 
Table 2. Observed wavenumbers ( $\tilde{v})$ of polarized Raman bands of $\alpha$-poly(L-alanine)

\begin{tabular}{|c|c|c|c|c|c|}
\hline \multicolumn{3}{|c|}{$\alpha$-PLA-NH ${ }^{a}$} & \multicolumn{3}{|c|}{$\alpha$-PLA-ND } \\
\hline $\begin{array}{c}A \\
\tilde{v} / \mathrm{cm}^{-1}\end{array}$ & $\begin{array}{c}E_{1} \\
\tilde{v} / \mathrm{cm}-1\end{array}$ & $\begin{array}{c}E_{2} \\
\tilde{v} / \mathrm{cm}^{-1}\end{array}$ & $\begin{array}{c}A \\
\tilde{v} / \mathrm{cm}^{-1}\end{array}$ & $\begin{array}{c}E_{1} \\
\tilde{v} / \mathrm{cm}^{-1}\end{array}$ & $\begin{array}{c}E_{2} \\
\tilde{v} / \mathrm{cm}^{-1}\end{array}$ \\
\hline \multicolumn{6}{|l|}{$3311 \mathrm{~ms}$} \\
\hline \multicolumn{6}{|l|}{$3288 m$} \\
\hline \multirow[t]{2}{*}{$2989 s$} & $2989 s$ & $2988 \mathrm{~s}$ & & & \\
\hline & 2970sh & & & & \\
\hline 2941 vs & 2941s & $2941 \mathrm{~s}$ & & & \\
\hline 2932sh & 2928sh & $2931 \mathrm{sh}$ & & & \\
\hline 2925sh & 2926sh & 2924sh & & & \\
\hline $2882 m$ & $2882 m$ & $2881 \mathrm{~m}$ & & & \\
\hline $2747 \mathrm{mw}$ & $2747 w$ & $2745 w$ & & & \\
\hline $1665 \mathrm{sh}$ & 1663sh & $1665 \mathrm{mw}$ & 1662sh & $1659 \mathrm{~ms}$ & 1656sh \\
\hline $1656 \mathrm{vs}$ & $1656 \mathrm{~ms}$ & $1655 \mathrm{~ms}$ & $1647 v s$ & $1647 \mathrm{~ms}$ & $1649 m$ \\
\hline $1555 v w$ & & & $1556 \mathrm{vw}$ & & \\
\hline 1465 sh & & & 1472sh & 1471sh & $1471 \mathrm{sh}$ \\
\hline \multirow{4}{*}{$1460 \mathrm{~ms}$} & $1456 \mathrm{~ms}$ & 1460sh & $1461 \mathrm{~ms}$ & $1457 s$ & \\
\hline & $1454 \mathrm{sh}$ & $1454 \mathrm{~s}$ & 1453sh & & $1455 \mathrm{~s}$ \\
\hline & & & & 1444sh & \\
\hline & & & 1429sh & & $1429 w^{b}$ \\
\hline \multirow[t]{3}{*}{$1381 \mathrm{mw}$} & $1381 w$ & $1382 w$ & $1379 \mathrm{mw}$ & $1381 w$ & $1382 \mathrm{mw}$ \\
\hline & $1338 \mathrm{sh}$ & $1337 \mathrm{~m}$ & & & \\
\hline & 1334sh & 1335sh & & & \\
\hline \multirow[t]{3}{*}{$1331 \mathrm{~s}$} & $1331 \mathrm{~m}$ & & $1327 \mathrm{~s}$ & $1327 \mathrm{~ms}$ & 1327sh \\
\hline & & & & $1325 \mathrm{sh}$ & $1324 \mathrm{~ms}$ \\
\hline & 1313sh & 1313sh & & & \\
\hline \multirow[t]{3}{*}{$1306 \mathrm{~s}$} & $1306 \mathrm{~m}$ & $1306 \mathrm{mw}$ & $1305 w^{c}$ & $1305 v w^{c}$ & \\
\hline & & & $1296 \mathrm{~m}$ & $1296 \mathrm{~m}$ & $1296 \mathrm{~m}$ \\
\hline & $1284 \mathrm{sh}$ & $1285 \mathrm{mw}$ & & & \\
\hline $1274 \mathrm{mw}$ & $1275 \mathrm{mw}$ & $1276 w$ & & & \\
\hline $1264 \mathrm{mw}$ & $1262 v w$ & $1262 \mathrm{mw}$ & & & \\
\hline $1242 w$ & $1242 w$ & $1243 w$ & $1239 v w$ & & \\
\hline $1229 w$ & $1229 v w$ & $1229 w$ & $1229 v w$ & & \\
\hline \multirow[t]{3}{*}{$1167 \mathrm{~ms}$} & $1168 \mathrm{mw}$ & $1166 \mathrm{mw}$ & $1171 \mathrm{~m}$ & $1169 w$ & $1170 w$ \\
\hline & & & & 1142sh & \\
\hline & & & $1134 \mathrm{~m}$ & $1134 w$ & $1135 w$ \\
\hline $1106 s$ & $1108 \mathrm{~ms}$ & $1106 s$ & $1107 m^{c}$ & $1107 m^{c}$ & $1107 \mathrm{mw}^{\mathrm{c}}$ \\
\hline $1094 w$ & 1094 sh & $1095 \mathrm{sh}$ & $1095 \mathrm{mw}$ & $1094 v w$ & $1094 w$ \\
\hline $1070 v w$ & $1071 w$ & $1070 \mathrm{mw}$ & & & \\
\hline $1051 w$ & $1051 w$ & $1051 \mathrm{sh}$ & $1062 \mathrm{mw}$ & $1062 \mathrm{mw}$ & $1062 \mathrm{mw}$ \\
\hline \multirow[t]{4}{*}{$1015 w$} & $1015 v w$ & $1014 w$ & $1017 v w$ & & $1021 \mathrm{vw}$ \\
\hline & & & $1001 \mathrm{~ms}$ & $999 \mathrm{mw}$ & $1000 \mathrm{~ms}$ \\
\hline & & & $975 \mathrm{mw}$ & $973 m$ & 973sh \\
\hline & & & 970sh & 970sh & $968 \mathrm{~m}$ \\
\hline $966 \mathrm{mw}$ & $964 w$ & $962 w$ & $946 \mathrm{~s}$ & $946 \mathrm{~m}$ & $946 \mathrm{~ms}$ \\
\hline $942 v w$ & $942 \mathrm{vw}$ & & & $936 \mathrm{sh}$ & \\
\hline 927sh & $928 \mathrm{vw}$ & $930 \mathrm{mw}$ & 922sh & & $922 w$ \\
\hline $908 v v s$ & $908 s$ & $908 s$ & $900 \mathrm{sh}^{\mathrm{c}}$ & $900 \mathrm{sh}^{\mathrm{c}}$ & $903 \mathrm{sh}^{\mathrm{c}}$ \\
\hline & 892sh & & & & \\
\hline & $882 w$ & $882 \mathrm{mw}$ & $888 \mathrm{vvs}$ & $888 s$ & $889 \mathrm{~ms}$ \\
\hline & & & & 878sh & 878sh \\
\hline $773 w$ & $771 v w$ & $770 w$ & $763 \mathrm{sh}$ & $764 w$ & $763 w$ \\
\hline $752 \mathrm{mw}$ & $753 w$ & $753 \mathrm{mw}^{\mathrm{b}}$ & $751 w$ & $753 \mathrm{mw}$ & $753 m w^{b}$ \\
\hline $693 \mathrm{mw}$ & $693 w$ & $693 \mathrm{mw}^{\mathrm{b}}$ & $685 w$ & $685 \mathrm{mw}$ & $685 \mathrm{~m}^{\mathrm{b}}$ \\
\hline $659 w$ & $661 w$ & $661 \mathrm{mw}$ & $653 w$ & $654 w$ & $655 \mathrm{~m}$ \\
\hline $638 \mathrm{mw}$ & & & & & \\
\hline $530 \mathrm{vs}$ & $530 \mathrm{~ms}$ & $530 \mathrm{~s}$ & $524 v s$ & $525 v s$ & 525vs \\
\hline $375 m$ & $374 m$ & $374 \mathrm{~ms}$ & $374 m$ & $374 \mathrm{~ms}$ & $374 \mathrm{~ms}$ \\
\hline & & & $363 \mathrm{sh}$ & $365 \mathrm{sh}$ & $365 \mathrm{sh}$ \\
\hline $329 \mathrm{mw}$ & $329 w$ & $328 \mathrm{vw}$ & $326 w$ & $326 w$ & $326 w$ \\
\hline $309 w$ & $311 \mathrm{mw}$ & $311 \mathrm{~m}$ & $309 w$ & $308 \mathrm{mw}$ & $308 \mathrm{~ms}$ \\
\hline $290 v w$ & $289 w$ & $290 \mathrm{mw}^{\mathrm{b}}$ & $287 w$ & $287 \mathrm{mw}$ & $289 m^{b}$ \\
\hline $263 \mathrm{~ms}$ & $260 \mathrm{mw}$ & $261 w$ & $262 \mathrm{~ms}$ & $260 \mathrm{mw}$ & $259 \mathrm{mw}$ \\
\hline $208 v w$ & $208 w$ & $209 m$ & $208 w$ & $207 \mathrm{mw}$ & $207 \mathrm{~m}$ \\
\hline $189 m$ & $189 \mathrm{~m}$ & $188 \mathrm{~m}$ & $187 \mathrm{~m}$ & $187 \mathrm{~ms}$ & $187 \mathrm{~m}$ \\
\hline $163 w$ & $163 \mathrm{~m}$ & $162 \mathrm{~m}$ & $162 w$ & $162 \mathrm{~m}$ & $161 \mathrm{~m}$ \\
\hline 139sh & & & 139sh & & \\
\hline 119sh & $119 \mathrm{mw}$ & 119sh & $120 \mathrm{~m}$ & $119 m$ & $118 w$ \\
\hline & $86 \mathrm{mw}$ & $84 \mathrm{~m}$ & $85 v w$ & $86 \mathrm{~ms}$ & $86 \mathrm{~ms}$ \\
\hline & 60sh & 60sh & & 60 sh & 60 sh \\
\hline $\begin{array}{l}\mathrm{s}=\text { Strong } \\
\text { ecommend } \\
\text { Raman spec } \\
\text { The intens } \\
\text { rientation } \\
\text { o the } E_{2} \mathrm{sp} \\
\text { Band due }\end{array}$ & $\begin{array}{l}\text { dium; w = } \\
\text { within one } \\
\text { damentals ar } \\
\text { s band seen } \\
\text { helix axis } \\
\text { d in some ca } \\
\text { erfect } N \text {-de }\end{array}$ & $\begin{array}{l}\text { = very; and } \\
\text { try species. } \\
\text { cs. } \\
\text { se mostly fro } \\
\text {. After trials } \\
\text { observed inf }\end{array}$ & $\begin{array}{l}\text { oulder. The } \\
\text { enumber vo } \\
\text { compone } \\
\text { pand was becies ban }\end{array}$ & $\begin{array}{l}\text { y comparis } \\
\text { the clearly } \\
\text { than from } \\
\text { gned to the } \\
\text { supported } t\end{array}$ & $\begin{array}{l}\text { een bands i } \\
\text { le (only from } \\
\text { o the random } \\
\text { es rather thar } \\
\text { inment. }\end{array}$ \\
\hline
\end{tabular}


$\mathrm{cm}^{-1}$ band is another that is clearly an $A$-species band from the infrared spectrum, ${ }^{19,22}$ so its relative strength in the $E_{2}$ spectrum may also be due to a large $\alpha_{11}$ component.

\section{CONCLUSIONS}

A complete vibrational analysis of $\alpha$-PLA, including a convincing normal mode analysis, requires experimental assignments of the observed infrared and Raman bands, i.e. the determination of the symmetry species to which each observed mode belongs. For the general regular helix, the infrared- and Raman-active $A$ and $E_{1}$ species are readily assigned from polarized infrared spectra of oriented samples. The $E_{2}$-species modes, however, are optically active only in the Raman spectra. In this study we have improved on previous work and present a more definitive experimental assignment of most of the $E_{2}$-species Raman bands.

The keys to achieving this assignment were twofold: obtaining more highly oriented samples and analysing the sources of 'cross-talk' between the spectra. In the first case, we believe we have obtained better sample orientation by refining the techniques for stroking out cast films. The resulting polarized laser Raman spectra give evidence of good chain orientation. In the second case, we have carefully examined factors affecting selection rules, such as reflection of the incident beam and non-perfect lateral and axial chain orientation in the sample, that lead to bands of one species appearing in spectra of another. This has permitted us to make secure assignments despite some 'cross-talk' between the spectra.

The new $E_{2}$-species assignments, plus polarized infrared and far-infrared spectra, ${ }^{22}$ have permitted the refinement of a new empirical force field for $\alpha$-PLA and a more complete normal mode analysis of this structure. $^{22}$

\section{Acknowledgement}

This research was supported by NSF grants MCB-9115906 and DMR-911053.

\section{REFERENCES}

1. A. Elliott, Proc. R. Soc., London, Ser. A 226, 408 (1954).

2. C. H. Bamford, A. Elliott and W. E. Hanby, Synthetic Polypeptides. Academic Press, New York (1956).

3. J. F. Rabolt, W. H. Moore and S. Krimm, Macromolecules 10 1065 (1977).

4. T. Miyazawa, K. Fukushima, S. Sugano and Y. Masuda, in Conformation of Biopolymers, edited by G. N. Ramachandran Vol. II, pp. 557-568. Academic Press, New York (1967).

5. K. Itoh, T. Nakahara, T. Shimanouchi, M. Oya, K. Uno and Y Iwakura, Biopolymers 6, 1759 (1968).

6. K. Itoh, T. Shimanouchi and M. Oya, Biopolymers 7, 649 (1969).

7. Y. Masuda, K. Fukushima, T. Fujii and T. Miyazawa, Biopolymers 8, 91 (1969).

8. K. Itoh and T. Shimanouchi, Biopolymers 9, 383 (1970).

9. B. Fanconi, B. Tomlinson, L. A. Nafie, W. Small and W. L. Peticolas, J. Chem. Phys. 51, 3993 (1969).

10. J. L. Koenig and P. L. Sutton, Biopolymers 8, 167 (1969).

11. L. Simons, G. Bergström, G. Blomfelt, S. Forss, H. Stenbäck and G. Wansén, Commentat. Phys.-Math. Soc. Sci. Fenn. 42 125 (1972).
12. B. Fanconi, Biopolymers 12, 2759 (1973)

13. B. G. Frushour and J. L. Koenig, Biopolymers 13, 455 (1974).

14. M. C. Chen and R. C. Lord, J. Am. Chem. Soc. 96, 4750 (1974).

15. M. Tipping, K. Viras and T. A. King, Biopolymers 23, 2891 (1984).

16. K. Itoh and T. Shimanouchi, Biopolymers 9, 383 (1970).

17. M. V. Krishnan and V. D. Gupta, Chem. Phys. Lett. 6, 231 (1970).

18. B. Fanconi, E. W. Small and W. L. Peticolas, Biopolymers 10, 1277 (1971).

19. A. M. Dwivedi and S. Krimm, Biopolymers 23, 923 (1984)

20. P. W. Higgs, Proc. R. Soc. London, Ser. A 133, 472 (1953).

21. H. Tadokoro, J. Chem. Phys. 33, 1558 (1960).

22. S. - H. Lee and S. Krimm, to be published.

23. L. N. Ovander, Opt. Spectrosc. 9, 302 (1960)

24. S.-H. Lee and S. Krimm, to be published.

25. R. S. Snyder, S. L. Hsu and S. Krimm, Spectrochim. Acta, Part A 34, 395 (1978) 